\title{
MtDNA variations at C5178a and A249d decrease the risk of severe COVID-19 in a Han Chinese population from Central China
}

\section{Yi Wu}

Hubei University of Medicine

\section{Xianhui Wang}

Hubei University of Medicine

Xihua Li

Hubei University of Medicine

Liyuan Song

Hubei University of Medicine

Shilong Yu

Army Medical University

\section{Zhicheng Fang}

Hubei University of Medicine

Yuquan Liu

Hubei University of Medicine

Leyong Yuan

Hubei University of Medicine

\section{Chunyan Peng}

Hubei University of Medicine

\section{Shenyi Zhang}

Hubei University of Medicine

\section{Wang Cheng}

Hubei University of Medicine

Hongchao Ma

Hubei University of Medicine

\section{Lifeng Wang}

Hubei University of Medicine

Junming Tang

Hubei University of Medicine

\section{Yunfu Wang}

Hubei University of Medicine

Fu-yun Ji ( $\sigma$ jifuyun@263.net )

Hubei University of Medicine https://orcid.org/0000-0002-5026-1861

\section{Research}

Keywords: mtDNA variations, SARS-CoV-2, COVID-19, risk, Han Chinese

Posted Date: March 6th, 2021 
DOI: https://doi.org/10.21203/rs.3.rs-285889/v1

License: (c) (i) This work is licensed under a Creative Commons Attribution 4.0 International License. Read Full License 


\section{Abstract}

Background: Mitochondria have been shown to play vital roles during SARS-CoV-2 infection and COVID-19 development. Currently, whether mitochondrial DNA (mtDNA) variations, which define mtDNA haplogroups and determine OXPHOS performance and ROS production, are associated with COVID-19 risk is unclear.

Methods: A population-based case-control study was conducted to compare the distribution of mtDNA variations defining mtDNA haplogroups between healthy controls $(n=615)$ and COVID-19 patients $(n=536)$. COVID-19 patients were diagnosed based on molecular diagnostics of the viral genome by qPCR and chest X-ray or computed tomography (CT) scanning. The exclusion criteria for the healthy controls were any history of diseases in the one-month preceding study assessment. MtDNA variations defining mtDNA haplogroups were identified by PCR-RFLPs and HVS-I sequencing and determined based upon mtDNA phylogenetic analysis using Mitomap Phylogeny. Student's t-test was used for continuous variables, and Pearson's chi-squared test or Fisher's exact test was used for categorical variables. To assess the independent effect of each mtDNA variation defining mtDNA haplogroups, multivariate logistic regression analyses were performed to calculate the adjusted odds ratios (ORs) and 95\% confidence intervals (Cls) with adjustments for the possible confounding factors of age, sex, smoking and diseases (including cardiopulmonary diseases, diabetes, obesity and hypertension) determined through clinical and radiographic examinations.

Results: Multivariate logistic regression analyses revealed that mtDNA variations at C5178a and A249d were associated with a reduced risk of severe COVID-19 $(\mathrm{OR}=0.590,95 \% \mathrm{Cl}=0.428-0.814, p=0.001$; and $\mathrm{OR}=0.654,95 \% \mathrm{Cl}=0.457-0.936, p=$ 0.020, respectively), while A4833G, A4715G, T3394C and G5417A/C16257a/C16261T were related to an increased risk of severe COVID-19 $(\mathrm{OR}=2.336,95 \% \mathrm{Cl}=1.179-4.608, p=0.015 ; \mathrm{OR}=2.033,95 \% \mathrm{Cl}=1.242-3.322, p=0.005 ; \mathrm{OR}=3.040,95 \%$ $\mathrm{Cl}=1.522-6.061, p=0.002 ;$ and $\mathrm{OR}=2.890,95 \% \mathrm{Cl}=1.199-6.993, p=0.018$, respectively).

Conclusion: mtDNA variations C5178a and A249d might contribute to an individual's resistance to developing severe COVID19, whereas A4833G, A4715G, T3394C and G5417A/C16257a/C16261T might increase an individual's risk of developing severe COVID-19.

\section{Trial registration: no.}

\section{Introduction}

The COVID-19 pandemic caused by SARS-CoV-2 has resulted in a worldwide crisis of formidable morbidity and mortality. The epidemiology, diagnosis, risk factors and treatments of COVID-19 have been explored intensively since the outbreak in Wuhan (Hubei Province, China) in December 2019. Many studies show that the clinical features of COVID-19 range from an asymptomatic state to acute respiratory distress syndrome (ARDS) and multiorgan dysfunction. Most COVID-19 patients develop a respiratory tract infection with common symptoms of cough, fever and shortness of breath. Other reported symptoms are weakness, malaise, respiratory distress, muscle pain, sore throat and loss of taste and/or smell. A number of patients develop severe fatal consequences resulting from a surge of inflammatory events (also known as cytokine storms) $[1,2]$. These clinical characteristics hint that although SARS-CoV-2 infection is the causative factor of COVID-19, not all of those exposed to SARS-CoV-2 will develop COVID-19, especially severe COVID-19, strongly suggesting that gene-environment interactions exist in COVID-19 progression, and an individual's hereditary susceptibility and innate capacities of antioxidant and immune responses to SARS-CoV-2 might contribute to this process. Currently, advanced age; male sex; blood group A; comorbidities of cardiopulmonary diseases, diabetes, obesity and hypertension [3]; a genomic segment of $\sim 50$ kb inherited from Neanderthals and carried by $~ 50 \%$ of people in South Asia and 16\% of people in Europe today [4]; and a 3p21.31 gene cluster have been reported to be related to an individual's susceptibility to severe COVID-19 [5]. Whether there are other specific molecular markers to predict the risk of COVID-19 remains unclear.

Recently, mitochondria have been inferred to be interrelated and interact with oxidative stress and inflammation during SARSCoV-2 infection and COVID-19 progression [6,7]. Furthermore, mitochondria have been shown to be indispensable regulators 
of innate and adaptive immune responses [8] and activation, development, maintenance and survival of the specific phenotypes of immune cells [9]. These findings provide clues that mitochondria might be associated with an individual's susceptibility to COVID-19.

As hubs of cellular oxidative homeostasis, mitochondria generate approximately $85 \%$ of intracellular reactive oxygen species (ROS) when they produce usable energy through oxidative phosphorylation (OXPHOS). In contrast to other cellular organelles, mitochondria have their own DNA (mtDNA). Interestingly, the common and "nonpathological" mtDNA variations defining mtDNA haplogroups determine OXPHOS performance and ROS production in humans and mice [10]. Additionally, these mtDNA variations exert considerable influence on longevity [11], help human beings adapt to different environments [12,13] and are associated with susceptibility to many human diseases in conditions where ROS generated by mitochondria are supposed to play a part [14-17]. Thus, we hypothesized that certain mtDNA variations defining mtDNA haplogroups might be related to an individual's susceptibility to COVID-19. To test this hypothesis, a population-based case-control study was performed to compare the distribution of mtDNA variations defining mtDNA haplogroups between COVID-19 patients and healthy controls in a Han Chinese population from Central China.

\section{Materials And Methods}

\section{Study population}

The study was approved by the Ethics Committee of Hubei University of Medicine and Wuhan University (Hubei Province, China). COVID-19 patients $(n=536)$ were recruited from Taihe Hospital (the First Affiliated Hospital of Hubei University of Medicine, Shiyan, Hubei Province, China) and People's Hospital of Hubei Province (the Affiliated Hospital of Wuhan University, Wuhan, Hubei Province, China) from February 2020 to March 2020. COVID-19 patients were diagnosed based on molecular diagnostics of the viral genome by qPCR and chest X-ray or computed tomography (CT) scanning. Age- and sex-matched volunteers $(n=615)$ were recruited from healthy individuals who underwent physical examinations at the two hospitals. The exclusion criteria for the healthy controls were any history of diseases in the one-month preceding study assessment. All the subjects were unrelated for at least three generations. After explaining the purpose and procedures of the study, all the participants signed a written informed consent form and completed a detailed questionnaire on smoking habits. Three milliliters of peripheral blood from each subject were drawn into Na-EDTA tubes. After incubation at $55^{\circ} \mathrm{C}$ for $30 \mathrm{~min}$ to inactivate the potential SARS-CoV-2, the blood samples were stored at $-80^{\circ} \mathrm{C}$ prior to genomic DNA extraction.

\section{Detection of mtDNA variations defining mtDNA haplogroups}

Genomic DNA was extracted from peripheral blood using an Ezup Column Blood Genomic DNA Purification Kit (Lot\#: B518253-0100, Sangon Biotech Co., Ltd, Shanghai, China). MtDNA variations defining mtDNA haplogroups were identified as described $[13,14,16,17]$. Briefly, after the entire mtDNA was amplified into 22 overlapping PCR fragments, the PCR fragments were digested with different restriction endonucleases (PCR-restriction fragment length polymorphism, PCR-RFLP) and replenished by sequencing hypervariable segment I (HVS-I). Two $\times$ Taq Plus Master Mix II was used for PCR-RFLP and HVS-I sequencing (Lot\#: P213-01, Vazyme Co., Ltd, Nanjing, China). The restriction endonucleases Alu I, Ava II, Bam HI, Bst NI, Dde I, Hae II, Hae III, Hha I, Hinc II, and Hinf I were used in the study (Takara Co., Ltd, Dalian, China). The primers for PCR-RFLPs and HVS-I sequencing were synthesized by Sangon Biotech Co., Ltd. (Shanghai, China), and the primer sequences are presented in Supplementary Table 1. MtDNA variations defining mtDNA haplogroups were determined based upon mtDNA phylogenetic analysis using Mitomap Phylogeny [12]. The Human Genome Variation Society (HGVS) validation of mtDNA variations is presented in Supplementary Table 2.

\section{Data analysis}

Student's $t$-test was used for continuous variables, and Pearson's chi-squared test or Fisher's exact test was used for categorical variables. For multiple comparisons of mtDNA variations defining mtDNA haplogroups, Bonferroni correction was applied (the required significance level $=0.05$ /number of comparisons). To assess the independent effect of each mtDNA 
variation defining mtDNA haplogroups, multivariate logistic regression analyses were performed to calculate the adjusted odds ratios (ORs) and 95\% confidence intervals (Cls) with adjustments for the possible confounding factors of age, sex, smoking and diseases (including cardiopulmonary diseases, diabetes, obesity and hypertension) determined through clinical and radiographic examinations. All statistical analyses were performed using SPSS Statistics 25 for Mac (SPSS Inc., Chicago, IL, USA).

\section{Results}

\section{MtDNA variations defining mtDNA haplogroups in controls and pooled cases}

In total, 536 unrelated COVID-19 patients and 615 unrelated healthy controls were recruited in the study. As shown in Table 1, the COVID-19 patients smoked more cigarettes $(p<0.001)$. MtDNA variations defining mtDNA haplogroups were detected for all the subjects (COVID-19 patients and healthy controls). Pearson's chi-squared test or Fisher's exact test showed that mtDNA variations A4833G (defining mtDNA haplogroup G), A4715G (defining mtDNA haplogroup M8), T3394C (defining mtDNA haplogroup M9), and G5417A/C16257a/C16261T (defining mtDNA haplogroup N9a) were significantly higher ( $p=0.005$, $0.002,0.027$ and 0.003 , respectively), while mtDNA variations C5178a (defining haplogroups D; the letter "a" indicates nucleotide transversion) and A249d (defining haplogroups F; the letter " $d$ " indicates nucleotide deletion) were significantly lower in COVID-19 patients than in controls $(p=0.002$ and 0.004 , respectively). When Bonferroni correction was applied, mtDNA variations A4715G, G5417A/C16257a/C16261T and C5178a reached the required $p$ value of $<0.0033(0.05 / 15)$. Multivariate logistic regression analyses with adjustments for age, sex, smoking and diseases (including cardiopulmonary diseases, diabetes, obesity and hypertension) revealed that, based upon a $p$ value of $<0.05$, mtDNA variations A4833G, A4715G, T3394C and G5417A/C16257a/C16261T were associated with an increased risk of COVID-19 $(\mathrm{OR}=4.384,95 \% \mathrm{Cl}=$ 2.103-9.137, $p=0.000 ; \mathrm{OR}=1.876,95 \% \mathrm{Cl}=1.167-3.021, p=0.009 ; \mathrm{OR}=2.618,95 \% \mathrm{Cl}=1.339-5.128, p=0.005 ;$ and $\mathrm{OR}=$ $3.401,95 \% \mathrm{Cl}=1.486-7.752, p=0.004$, respectively). In contrast, the $\mathrm{C} 5178 \mathrm{a}$ and $\mathrm{A} 249 \mathrm{~d} \mathrm{mtDNA}$ variations were associated with a reduced risk of COVID-19 $(\mathrm{OR}=0.681,95 \% \mathrm{Cl}=0.504-0.919, p=0.012$; and $\mathrm{OR}=0.639,95 \% \mathrm{Cl}=0.483-0.847, p=$ 0.002 , respectively) (Table 2 and Figure 1 ).

\section{Frequencies of mtDNA variations defining mtDNA haplogroups in controls and moderate cases}

When COVID-19 patients were stratified into cases with moderate (non-ICU) and severe (ICU) disease (patients with ARDS, multiple organ dysfunction, or metabolic acidosis), Pearson's chi-squared test or Fisher's exact test showed that mtDNA variations A663G (defining mtDNA haplogroup A) and G5417A/C16257a/C16261T were significantly higher $(p<0 r=0.001$, respectively), while mtDNA variation 8281-8289d (specific for mtDNA haplogroup $B$ ) was significantly lower in moderate COVID-19 patients than in controls $(p<0.001)$. All the mtDNA variations (A663G, G5417A/C16257a/C16261T and 8281$8289 \mathrm{~d}$ ) reached the required $p$ value of $<0.0033$ when Bonferroni correction was applied. Multivariate logistic regression analyses with adjustments for age, sex, smoking and diseases (including cardiopulmonary diseases, diabetes, obesity and hypertension) showed that mtDNA variation 8281-8289d was associated with a reduced risk of moderate COVID-19 (OR $=$ $0.034,95 \% \mathrm{Cl}=0.016-0.068, p=0.000$ ), whereas mtDNA variations A663G and G5417A/C16257a/C16261T were related to an increased risk of moderate COVID-19 $(\mathrm{OR}=2.445,95 \% \mathrm{Cl}=1.116-5.348, p=0.026$; and $\mathrm{OR}=3.984,95 \% \mathrm{Cl}=1.325-11.904$, $p=0.014$, respectively) (Table 3 and Figure 1 ).

\section{Distribution of mtDNA variations defining mtDNA haplogroups in controls and severe cases}

Pearson's chi-squared test or Fisher's exact test demonstrated that mtDNA variations A4833G, A4715G, T3394C and G5417A/C16257a/C16261T were significantly higher ( $p=0.003,0.001,0.010$, and 0.023 , respectively), while mtDNA variations C5178a and A249d were significantly lower in severe COVID-19 patients than in controls $(p=0.000$ and 0.008 , respectively). When Bonferroni correction was applied, mtDNA variations A4833G, A4715G and C5178a reached the required $p$ value of $<0.0033$. Multivariate logistic regression analyses with adjustments for age, sex, smoking and diseases (including cardiopulmonary diseases, diabetes, obesity and hypertension) showed that the C5178a and A249d mtDNA variations were associated with a reduced risk of severe COVID-19 $(\mathrm{OR}=0.590,95 \% \mathrm{Cl}=0.428-0.814, p=0.001$; and $\mathrm{OR}=0.654,95 \% \mathrm{Cl}=$ 
0.457-0.936, $p=0.020$ ), while the A4833G, A4715G, T3394C and G5417A/C16257a/C16261T mtDNA variations were related to an increased risk of severe COVID-19 $(\mathrm{OR}=2.336,95 \% \mathrm{Cl}=1.179-4.608, p=0.015 ; \mathrm{OR}=2.033,95 \% \mathrm{Cl}=1.242-3.322, p=$ $0.005 ; \mathrm{OR}=3.040,95 \% \mathrm{Cl}=1.522-6.061, p=0.002 ;$ and $\mathrm{OR}=2.890,95 \% \mathrm{Cl}=1.199-6.993, p=0.018$, respectively) (Table 4 and Figure 1).

\section{Discussion}

COVID-19 caused by SARS-CoV-2 has been a worldwide health emergency. To provide insight into COVID-19 risk from the point of mitochondria, we conducted a case-control study in a Han Chinese population from Central China. Our data demonstrated that mtDNA variations C5178a and A249d contributed to an individual's resistance to developing severe COVID19, whereas mtDNA variations A4833G, A4715G, T3394C and G5417A/C16257a/C16261T increased the risk of severe COVID-19 in this Han Chinese population. Additionally, mtDNA variations A663G and G5417A/C16257a/C16261T increased an individual's risk of developing moderate COVID-19, while the mtDNA variation 8281-8289d decreased an individual's risk of developing moderate COVID-19. MtDNA variations G5417A/C16257a/C16261T were risk factors for both moderate and severe COVID-19.

As hubs of cellular oxidative homeostasis, mitochondria are not only interrelated and play a role in the oxidative stress and inflammation in SARS-CoV-2 infection and COVID-19 development [6,7,18-21] but are also indispensable regulators of the innate and adaptive immune responses in the process of SARS-CoV-2 infection and COVID-19 development [8,9,21-23]. Moreover, mitochondrial residency of SARS-CoV-2 with a stronger signal compared to its coronavirus relatives implied further that mitochondria are the major cellular organelle affected by oxidative stress and inflammation resulting from SARS-CoV-2 infection [24]. In comparison with nuclear DNA (nDNA), mtDNA is particularly susceptible to oxidative damage due to its direct exposure to ROS, limited DNA repair capacity and absence of protection by histones. The decline in mitochondrial function with aging might explain the phenomena of high mortality in elderly COVID-19 patients to a certain extent $[1,11,15]$. Thus, when SARS-CoV-2 infects cells, the common and "nonpathological" mtDNA variants, which define mtDNA haplogroups and determine OXPHOS performance and ROS production, contribute to an individual's capacity for antioxidant and immune responses to protect cells from SARS-CoV-2 infection and COVID-19 development or aggravate the process. Consistent with this, mtDNA variation C5178a (L237M, Leu $\rightarrow$ Met substitution) in ND2, defining mtDNA haplogroup D and proposed to be an efficient oxidant scavenger [25], was significantly lower both in the pooled COVID-19 patients and severe COVID-19 patients compared to controls in the study. The protective effect of the C5178a mtDNA variation has been reported to be propitious to human longevity [11], beneficial for diabetic patients against atherosclerotic and myocardial infarction [26], and to decrease an individual's risk of developing acute mountain sickness (AMS), lung cancer, chronic obstructive pulmonary disease (COPD) and other diseases $[14-16,26]$. Therefore, the protective effect of the C5178a mtDNA variation against oxidative damage as an efficient oxidant scavenger might protect cells from oxidative destruction caused by SARS-CoV-2 infection and decrease an individual's risk of developing COVID-19, especially severe COVID-19.

MtDNA variations A249d, T6392C and G10310A are the common variants of mtDNA subhaplogroups F1-4 [27]. In the study, mtDNA variations G12406A (defining mtDNA subhaplogroup F1) and T16298C/C16304T/T16362C (defining mtDNA subhaplogroup F3) were detected, and both were significantly lower in COVID-19 patients ( $p=0.043$ and 0.018 , respectively). As expected, the combined subhaplogroups F1 and F3 (haplogroup F) were shown to be associated with a decreased risk of COVID-19 and severe COVID-19. In Asian populations, haplogroup F functions as a positive factor for a long-life span [28], confers beneficial effects to resist metabolic syndrome (MetS) [29], and improves the physical performance of athletes [30]. Because only subhaplogroups F1 and F3 were detected and mtDNA variations at T6392C and G10310A were synonymous, we deduced that variation at A249d in the control region (HVS-II) of mtDNA might account for its protective effect against COVID-19.

MtDNA variations A4833G, A4715G, T3394C and G5417A/C16257a/C16261T were found to increase an individual's risk of developing severe COVID-19 in the study. Of note, A4715G, T3394C and G5417A/C16257a/C16261T are reported to be associated with an increased risk of type II diabetes mellitus (T2DM) in the Chinese population [31]. A4715G is a risk factor 
for moderate and severe nonalcoholic fatty liver disease (NAFLD) [32]. The mtDNA variation T3394C is beneficial for native Tibetans to adapt to hypoxic environments because it has higher complex I activity [13]. However, in plain areas, T3394C increases an individual's risk of many diseases, including LHON [33], hypertension [34] and T2DM [35]. Additionally, T3394C is a candidate that counteracts longevity [36]. Similar to T3394C, A4833G was significantly higher in native Tibetans residing at high altitudes than in Han Chinese individuals living in plain areas $(32 / 289$ vs. 39/1605, $p=0.0001)$ [13], which might help native Tibetans adapt to hypoxic environments. Similarly, A4833G constitutes a risk factor for lung cancer [16], COPD [17] and recurrent oral ulceration (ROU) in plain areas [37]. Therefore, T3394C and A4833G might be risk factors for severe COVID-19 through the same mechanism as in the other human diseases occurring in the plain areas.

MtDNA variation G5417A (specific for mtDNA haplogroup N9) confers a higher risk of MetS development in HIV-infected patients [38]. In the Chinese population, the mtDNA variation G5417A/C16257a/C16261T is a risk factor for diabetic nephropathy because of having more ROS and fragmented mitochondria [31], which might account for it being a risk factor for both moderate and severe COVID-19 in this Han Chinese population.

Of note, a mtDNA deletion of approximately 800 bp was detected during PCR-RFLP analysis in the study. In our previous studies, an 822-bp mtDNA deletion was identified and demonstrated to be positively associated with cigarette smoking and mtDNA haplogroups $[16,17]$. Because the blood samples of the study were incubated at $55^{\circ} \mathrm{C}$ for 30 min to inactivate the potential SARS-CoV-2, the mtDNA deletion was not further analyzed to avoid the possible disturbance of mtDNA breakage caused by incubation at higher temperature.

This study has some limitations. First, during the extreme clinical circumstances of the pandemic, especially at the beginning of the epidemic, we were unable to collect detailed clinical data (for example, levels of inflammatory cytokines and immune factors and disease outcome) in a very short period of time, which will be important to investigate in follow-up studies.

Second, because we only analyzed mtDNA variations in a Han Chinese population from Central China, large-scale studies are expected in other populations.

\section{Conclusions}

Our findings revealed that mtDNA variations C5178a and A249d contribute to an individual's resistance to COVID-19 development, whereas variations A4833G, A4715G, T3394C, and G5417A/C16257a/C16261T might be risk factors for this process, providing evidence that gene-environment interactions do exist in COVID-19 progression.

\section{Declarations}

\section{Ethics approval and consent to participate}

The study was approved by the Ethics Committee of Hubei University of Medicine and Wuhan University (Hubei Province, China). All the participants signed a written informed consent form to participate the project.

\section{Consent for publication}

All the authors have reviewed the final version of manuscript and signed a written consent form for publication.

\section{Availability of data and materials}

The data that support the findings of this study are available from the corresponding author upon reasonable request.

\section{Competing interests}

The authors declare no conflict of interest.

\section{Acknowledgements}


Authors thank all the participants for their willingness to contribute to the understanding of COVID-19. Many thanks to Dr. Bin Qiao for his kind gift of DNA samples of COVID-19 patients (People's Hospital of Hubei Province, Wuhan University).

\section{Funding}

This work was supported by grants from the Special Project of Contingency Research for COVID-19 (No:2020XGFYZR11) and the Cultivating Project for Young Scholar at Hubei University of Medicine (No: 2018QDJZR01) awarded to Dr. Fuyun Ji; and the Special Project of Contingency Research for COVID-19 at Hubei University of Medicine (No:2020XGFYZR03) awarded to Dr. Yunfu Wang. The funders had no role in study design, in collection, analysis and interpretation of data, in writing of the manuscript, and in decision to submit the article for publication.

\section{Authors' contributions}

The author contributions were as follows: FYJ and YFW, concept and design; $Y W, X H W$ and $X H L$, performing the experiments; ZCF, CYP, YQL, LYY, LY, SYZ, WC, LFW and HCM, sample collection, storage and DNA isolation; XHW, XHL, FYJ, and SLY, data collection and analysis; FYJ and YFW, contributed reagents/materials/analysis tools; FYJ, YFW and JMT, writing of the paper. All the authors critically reviewed the manuscript and approved the final version for publication.

\section{References}

1. Huang CL, Wang YM, Li XW, Ren LL, Cao B. Clinical features of patients infected with 2019 novel coronavirus in Wuhan, China. Lancet. 2020;395(10223):497-506.

2. Wang CF, Xie J, Zhao L, Fei XC, Zhang H, Tan Y, et al. Alveolar macrophage dysfunction and cytokine storm in the pathogenesis of two severe COVID-19 patients. EBioMedicine. 2020; 57:102833.

3. Zhou F, Yu T, Du RH, Fan GH, Liu Y, Liu ZB, et al. Clinical course and risk factors for mortality of adult inpatients with COVID-19 in Wuhan, China: a retrospective cohort study. Lancet. 2020;395(10229):1054-1062.

4. Zeberg H, Paabo S. The major genetic risk factor for severe COVID-19 is inherited from Neanderthals. Nature. 2020;587(7835):610-612.

5. Severe Covid-19 GWAS Group, Ellinghaus D, Degenhardt F, Bujanda L, Buti M, Albillos A, et al. Genomewide association study of severe COVID-19 with respiratory failure. N Engl J Med. 2020;383(16):1522-1534.

6. Jumana S, Peyssonnaux C, Singh KK, Edeas M. Mitochondria and Microbiota dysfunction in COVID-19 pathogenesis. Mitochondrion. 2020; 54: 1-7.

7. Wang F, Nie JY, Wang HZ, Zhao Q, Zhang YX. Characteristics of Peripheral Lymphocyte Subset Alteration in COVID-19 Pneumonia. J Infect Dis. 2020;221(11):1762-1769.

8. Weinberg SE, Sena LA, Chandel NS. Mitochondria in the Regulation of Innate and Adaptive Immunity. Immunity. 2015;42(3):406-17.

9. Angajala A, Lim S, Phillips JB, Kim JH, Clayton Y, You ZB, et al. Diverse Roles of Mitochondria in Immune Responses: Novel Insights Into Immuno-Metabolism. Front Immunol. 2018; 9:1605.

10. Moreno-Loshuertos R, Acín-Pérez R, Fernández-Silva P, Movilla N, Pérez-Martos A, Rodriguez de Cordoba S, et al. Differences in reactive oxygen species production explain the phenotypes associated with common mouse mitochondrial DNA variants. Nat Genet. 2006;38(11):1261-8.

11. Tanaka M, Gong JS, Zhang J, Yoneda M, Yagi K. Mitochondrial genotype associated with longevity. Lancet. 1998;351(9097):185-6.

12. Mishmar D, Eduardo RP, Golik P, Macaulay V, Clark AG, Hosseini S, et al. Natural selection shaped regional mtDNA variation in humans. Proc Natl Acad Sci USA. 2003;100(1):171-6.

13. Ji F, Sharpley MS, Derbeneva O, Alves LS, Qian P, Wang Y, et al. Mitochondrial DNA variant associated with Leber hereditary optic neuropathy and high-altitude Tibetans. Proc Natl Acad Sci USA. 2012;109(19):7391-6. 
14. Li FX, Ji FY, Zheng SZ, Yao W, Qian GS. MtDNA haplogroups M7 and B in southwestern Han Chinese at risk for acute mountain sickness. Mitochondrion. 2011;11(4):553-8.

15. Wallace DC. A mitochondrial paradigm of metabolic and degenerative diseases, aging, and cancer: a dawn for evolutionary medicine. Annu Rev Genet. 2005; 39:359-407.

16. Zheng SZ, Qian P, Li FX, Qian GS, Wang CZ, Wu GM, et al. Association of Mitochondrial DNA Variations with Lung Cancer Risk in a Han Chinese Population from Southwestern China. PLoS One. 2012;7(2): e31322.

17. Zheng SZ, Wang CZ, Qian GS, Wu GM, Guo RL, Li Q, et al. Role of mtDNA haplogroups in COPD susceptibility in a southwestern Han Chinese population. Free Radic Biol Med. 2012;53(3):473-81.

18. Markus H, Hannah KW, Simon S, Nadine K, Stefan P. SARS-CoV-2 Cell Entry Depends on ACE2 and TMPRSS2 and IS Blocked by a Clinically Proven Protease Inhibitor. Cell. 2020;181(2):271-280.e8.

19. Tian SF, Xiong Y, Liu H, Niu L, Guo JC, Liao MY, et al. Pathological study of the 2019 novel coronavirus disease (COVID19) through postmortem core biopsies. Mod Pathol. 2020;33(6):1007-1014.

20. Xiao HL, Zhao LX, Yang J, Tong N, An L, Liu QT, et al. Association between ACE2/ACE balance and pneumocyte apoptosis in a porcine model of acute pulmonary thromboembolism with cardiac arrest. Mol Med Rep. 2018;17(3):42214228.

21. Zhang D, Guo R, Lei L, Liu HJ, Wang YW, Wang YL, et al. COVID-19 infection induces readily detectable morphologic and inflammation- related phenotypic changes in peripheral blood monocytes. J Leukoc Biol. 2020;10.1002/JLB.4HI0720470R. doi: 10.1002/JLB.4HI0720-470R.Online ahead of print.

22. Hoffmann RF, Zarrintan S, Brandenburg SM, Kol A, de Bruin HG, Jafari S, et al. Prolonged cigarette smoke exposure alters mitochondrial structure and function in airway epithelial cells. Respir Res. 2013;14(1):97.

23. Hoffmann RF, Jonker MR, Brandenburg SM, de Bruin HG, Ten Hacken NHT, van Oosterhout AJM, et al. Mitochondrial dysfunction increases pro-inflammatory cytokine production and impairs repair and corticosteroid responsiveness in lung epithelium. Sci Rep. 2019; 9(1): 15047.

24. Wu KE, Zou J, Chang HY. RNA-GPS Predicts SARS-CoV-2 RNA Localization to Host Mitochondria and Nucleolus. Cell Syst. 2020;11(1):102-108.e3.

25. Levine RL, Mosoni L, Berlett BS, Stadtman ER. Methionine residues as endogenous antioxidants in proteins. Proc Natl Acad Sci USA. 1996;93(26):15036- 40.

26. Takagi K, Yamada YJ, Gong JS, Sone T, Yokota M, Tanaka M. Association of a 5178C $\rightarrow$ A (Leu237Met) polymorphism in the mitochondrial DNA with a low prevalence of myocardial infarction in Japanese individuals. Atherosclerosis. 2004;175(2):281-6.

27. Yao YG, Kong QP, Wang CY, Zhu CL, Zhang YP. Different matrilineal contributions to genetic structure of ethnic groups in the Silk Road region in China. Mol Biol Evol. 2004;21(12):2265-80.

28. Feng J, Zhang JY, Liu M, Wan G. Qi KY, Zheng CG, et al. Association of mtDNA haplogroup F with healthy longevity in the female Chuang population, China. Exp Gerontol. 2011;46(12):987-93.

29. Hu C, He X, Li X, Sun L, Zheng C, Liang Q, et al. Comparative study for the association of mitochondrial haplogroup F+ and metabolic syndrome between longevity and control population in Guangxi Zhuang Autonomous Region, China. J Nutr Health Aging. 2018;22(2):302-307.

30. InWook H, Kim K, Choi EJ, Han J. Association of mitochondrial haplogroup F with physical performance in Korean population. Genomics Inform. 2019;17(1): e11.

31. Fang HZ, Hu NQ, Zhao QY, Wang BQ, Zhou HB. mtDNA Haplogroup N9a Increases the Risk of Type 2 Diabetes by Altering Mitochondrial Function and Intracellular Mitochondrial Signals. Diabetes. 2018; 67(7): 1441-1453.

32. Lu MY, Huang JF, Liao YC, Bai RK, Trieu RB, Chuang WL, et al. Mitochondrial polymorphism 12361A>G is associated with nonalcoholic fatty liver disease. Transl Res. 2012;159(1):58-9. 
33. Nakaso K, Adachi Y, Fusayasu E, Doi K, Imamura K, Yasui K, et al. Leber's Hereditary Optic Neuropathy with Olivocerebellar Degeneration due to G11778A and T3394C Mutations in the Mitochondrial DNA. J Clin Neurol. 2012;8(3):230-4.

34. Shao JZ, Chen CG, Lin WH, Dong ZB, Xu JZ. Clinical and molecular features of a Han Chinese family with maternally transmitted hypertension. Int J Clin Exp Pathol. 2017;10(7):7384-7389.

35. Tang DL, Zhou X, Li X, Zhao L, Liu F. Variation of mitochondrial gene and the association with type 2 diabetes mellitus in a Chinese population. Diabetes Res Clin Pract. 2006;73(1):77-82.

36. Li L, Zheng HX, Liu ZY, Qin ZD, Chen F, Qian DG, et al. Mitochondrial genomes and exceptional longevity in a Chinese population: the Rugao longevity study. Age (Dordr). 2015;37(1):9750.

37. Sun M, Fu SM, Wang LF, Dong GY, Wu D. Hypervariable Region Polymorphism of mtDNA of Recurrent Oral Ulceration in Chinese. PLoS One. 2012;7(9): e45359.

38. Zhao D, Ding YY, Lin HJ, Chen XX, Shen WW. Mitochondrial Haplogroups N9 and G Are Associated with Metabolic Syndrome Among Human Immunodeficiency Virus-Infected Patients in China. AIDS Res Hum Retroviruses. 2019;35(6):536-543.

\section{Tables}

Table 1 Clinical characteristics of the study population

\begin{tabular}{|llll|}
\hline Characteristic & Controls $(\mathrm{n}=615)(\%)$ & Cases $(\mathrm{n}=536)(\%)$ & $p$ value \\
\hline Gender & $356(57.89)$ & $319(59.51)$ & $0.590^{\mathrm{a}}$ \\
\hline Male & $259(42.11)$ & $217(40.49)$ & \\
\hline Female & $58.29 \pm 13.87$ & $60.83 \pm 14.46$ & $0.052^{\mathrm{b}}$ \\
\hline$<39$ & $48(7.87)$ & $46(8.58)$ & \\
\hline $40-49$ & $106(17.24)$ & $82(15.30)$ & \\
\hline $50-59$ & $112(18.21)$ & $91(16.98)$ & \\
\hline $60-69$ & $217(35.28)$ & $186(34.70)$ & \\
\hline$>70$ & $132(21.46)$ & $131(24.44)$ & $0.098^{\mathrm{a}}$ \\
\hline Pack-years of smoking $(\%)^{c}$ & & & $<0.001^{\mathrm{b}}$ \\
\hline $0-20$ & $543(88.29)$ & $455(84.89)$ & \\
\hline$>20$ & $72(11.71)$ & $81(15.11)$ & $9.27 \pm 12.54$ \\
\hline Mean pack-years & $6.35 \pm 10.86$ & & \\
\hline
\end{tabular}

Data are presented as mean $\pm S D$, unless otherwise specified.

a,$\chi^{2}$-Test or Fisher's exact test.

$\mathrm{b}, t$-Test.

${ }^{\mathrm{c}}$, the median number of pack-year cigarette smoking of the pooled subjects of COVID-19 patients and healthy controls were utilized as the cut-off point to stratify the subjects ( 1 pack-year $=20$ cigarettes per day for 1 year). 
Table 2 The frequencies of mtDNA variations defining mtDNA haplogroups among controls and pooled cases

\begin{tabular}{|c|c|c|c|c|c|c|}
\hline MtDNA variations & $\begin{array}{l}\text { mtDNA } \\
\text { haplogroups }\end{array}$ & $\begin{array}{l}\text { Controls (n } \\
=615)(\%)\end{array}$ & $\begin{array}{l}\text { Cases }(n= \\
536)(\%)\end{array}$ & $\begin{array}{l}p \\
\text { value } \\
\left(\chi^{2}\right)^{a}\end{array}$ & $\begin{array}{l}\text { Adjusted } \\
p \text { value }^{\mathrm{b}}\end{array}$ & $\begin{array}{l}\text { OR }(95 \% \\
\mathrm{Cl})^{b}\end{array}$ \\
\hline A663G & A & $39(6.34)$ & $48(8.96)$ & 0.117 & 0.060 & $\begin{array}{l}1.567 \\
(0.980- \\
2.500)\end{array}$ \\
\hline $8281-8289 d^{c}$ & B & 118 (19.19) & $87(16.23)$ & 0.217 & 0.150 & $\begin{array}{l}0.781 \\
(0.557- \\
1.093)\end{array}$ \\
\hline $\mathrm{C} 5178 \mathrm{a}^{\mathrm{c}}$ & $\mathrm{D}$ & $176(28.62)$ & $\begin{array}{l}110 \\
(20.52)\end{array}$ & 0.002 & 0.012 & $\begin{array}{l}0.681 \\
(0.504- \\
0.919)\end{array}$ \\
\hline A249d/T6392C/G10310A & $F(F 1+F 3)$ & $129(20.98)$ & $77(14.37)$ & 0.004 & 0.002 & $\begin{array}{l}0.639 \\
(0.483- \\
0.847)\end{array}$ \\
\hline A4833G & G & $16(2.60)$ & $32(5.97)$ & 0.005 & 0.000 & $\begin{array}{l}4.384 \\
(2.103- \\
9.137)\end{array}$ \\
\hline T9824C & M7 & $33(5.37)$ & $41(7.65)$ & 0.119 & 0.519 & $\begin{array}{l}1.181 \\
(0.712- \\
1.957)\end{array}$ \\
\hline A4715G & $\begin{array}{l}\text { M8 (M8a + } \\
C+Z)\end{array}$ & $36(5.85)$ & $59(11.01)$ & 0.002 & 0.009 & $\begin{array}{l}1.876 \\
(1.167- \\
3.021)\end{array}$ \\
\hline T3394C & M9 & $18(2.93)$ & $30(5.60)$ & 0.027 & 0.005 & $\begin{array}{l}2.618 \\
(1.339- \\
5.128)\end{array}$ \\
\hline G16274A & M2 & $8(1.30)$ & $5(0.93)$ & 0.399 & 0.896 & $\begin{array}{l}0.899 \\
(0.180- \\
4.484)\end{array}$ \\
\hline T16126C & M3 & $10(1.63)$ & $7(1.30)$ & 0.616 & 0.466 & $\begin{array}{l}0.572 \\
(0.128- \\
2.567)\end{array}$ \\
\hline T16311C & M10 & $9(1.46)$ & $6(1.12)$ & 0.592 & 0.418 & $\begin{array}{l}0.509 \\
(0.099- \\
2.610)\end{array}$ \\
\hline G6023A & M13 & $4(0.65)$ & $4(0.75)$ & 1.000 & 0.514 & $\begin{array}{l}0.566 \\
(0.102- \\
3.137)\end{array}$ \\
\hline T489C/C10400T/T14783C/G15043A & $M^{*}$ & $1(0.16)$ & $1(0.19)$ & 1.000 & NA & \\
\hline G5417A/C16257a/C16261T & N9a & $11(1.79)$ & $27(5.04)$ & 0.003 & 0.004 & $\begin{array}{l}3.401 \\
(1.486- \\
7.752)\end{array}$ \\
\hline T1391C/T16311C & $\mathrm{R} 1$ & $7(1.14)$ & $2(0.37)$ & 0.187 & 0.219 & $\begin{array}{l}0.353 \\
(0.067- \\
1.859)\end{array}$ \\
\hline
\end{tabular}

a, $\chi^{2}$-Test or Fisher's exact test. Bonferroni corrected $p<0.05 / n(n=15)$. 
b, $p$ value and ORs ( $95 \% \mathrm{Cls}$ ) determined by multivariate logistic regression analysis, adjusted for age, gender, smoking and diseases (including cardiopulmonary diseases, diabetes, obesity and hypertension determined through clinical and radiographic examinations).

c, the letter "d" indicates nucleotide deletion, the letter "a" indicates nucleotide transversion.

Table 3 The distribution of mtDNA variations defining mtDNA haplogroups among controls and moderate cases

\begin{tabular}{|c|c|c|c|c|c|c|}
\hline MtDNA variations & mtDNA haplogroups & $\begin{array}{l}\text { Controls } \\
(n= \\
615)(\%)\end{array}$ & $\begin{array}{l}\text { Cases } \\
(n=85) \\
(\%)\end{array}$ & $\begin{array}{l}p \\
\text { value } \\
\left(\chi^{2}\right)^{a}\end{array}$ & $\begin{array}{l}\text { Adjusted } \\
p \text { value }^{\mathrm{b}}\end{array}$ & $\begin{array}{l}\text { OR } \\
(95 \% \mathrm{Cl}) \\
\mathrm{b}\end{array}$ \\
\hline A663G & A & $\begin{array}{l}39 \\
(6.34)\end{array}$ & $\begin{array}{l}10 \\
(11.76)\end{array}$ & $<.001$ & 0.026 & $\begin{array}{l}2.445 \\
(1.116- \\
5.348)\end{array}$ \\
\hline $8281-8289 d^{C}$ & B & $\begin{array}{l}118 \\
(19.19)\end{array}$ & $\begin{array}{l}9 \\
(10.59)\end{array}$ & $<.001$ & 0.000 & $\begin{array}{l}0.034 \\
(0.016- \\
0.068)\end{array}$ \\
\hline $\mathrm{C} 5178 \mathrm{a}^{\mathrm{C}}$ & $\mathrm{D}$ & $\begin{array}{l}176 \\
(28.62)\end{array}$ & $\begin{array}{l}25 \\
(29.41)\end{array}$ & 0.898 & 0.362 & $\begin{array}{l}1.289 \\
(0.747- \\
2.222)\end{array}$ \\
\hline A249d/T6392C/G10310A & $\mathrm{F}$ & $\begin{array}{l}129 \\
(20.98)\end{array}$ & $\begin{array}{l}11 \\
(12.94)\end{array}$ & 0.110 & 0.092 & $\begin{array}{l}1.969 \\
(1.896- \\
4.326)\end{array}$ \\
\hline A4833G & G & $\begin{array}{l}16 \\
(2.60)\end{array}$ & $3(3.53)$ & 0.494 & 0.712 & $\begin{array}{l}1.182 \\
(0.486- \\
2.875)\end{array}$ \\
\hline T9824C & M7 & $\begin{array}{l}33 \\
(5.37)\end{array}$ & $5(5.88)$ & 1.000 & 0.765 & $\begin{array}{l}1.185 \\
(0.389- \\
3.610)\end{array}$ \\
\hline A4715G & M8 (M8a + C + Z) & $\begin{array}{l}36 \\
(5.85)\end{array}$ & $6(7.06)$ & 0.808 & 0.910 & $\begin{array}{l}1.449 \\
(0.700- \\
3.003)\end{array}$ \\
\hline T3394C & M9 & $\begin{array}{l}18 \\
(2.93)\end{array}$ & $2(2.35)$ & 1.000 & 0.903 & $\begin{array}{l}0.910 \\
(0.199- \\
4.167)\end{array}$ \\
\hline T489C/C10400T/T14783C/G15043A & $\begin{array}{l}\mathrm{M}^{*} \\
(\mathrm{M} 2+\mathrm{M} 3+\mathrm{M} 10+\mathrm{M} 13+\mathrm{M})\end{array}$ & $\begin{array}{l}32 \\
(5.20)\end{array}$ & $\begin{array}{l}6 \\
(7.06 \%)\end{array}$ & 0.446 & NA & \\
\hline G5417A/C16257a/C16261T & $\mathrm{N} 9 \mathrm{a}$ & $\begin{array}{l}11 \\
(1.79)\end{array}$ & $8(9.41)$ & 0.001 & 0.014 & $\begin{array}{l}3.984 \\
(1.325- \\
11.904)\end{array}$ \\
\hline T1391C/T16311C & $\mathrm{R} 1$ & $7(1.14)$ & $0(0.00)$ & 0.607 & 0.999 & $\begin{array}{l}0.000 \\
(0.000-.)\end{array}$ \\
\hline
\end{tabular}

a, $\chi^{2}$-Test or Fisher's exact test. Bonferroni corrected $p<0.05 / \mathrm{n}(\mathrm{n}=15)$.

b , adjusted $p$ value and ORs (95\% Cls) determined by multivariate logistic regression analysis, adjusted for age, gender, smoking and diseases (including cardiopulmonary diseases, diabetes, obesity and hypertension determined through clinical and radiographic examinations).

c, the letter "d" indicates nucleotide deletion, the letter "a" indicates nucleotide transversion.

Table 4 The distribution of mtDNA variations defining mtDNA haplogroups among controls and severe cases 


\begin{tabular}{|c|c|c|c|c|c|c|}
\hline MtDNA variations & mtDNA haplogroups & $\begin{array}{l}\text { Controls } \\
(n= \\
615) \\
(\%)\end{array}$ & $\begin{array}{l}\text { Cases } \\
(\mathrm{n}= \\
451) \\
(\%)\end{array}$ & $\begin{array}{l}p \\
\text { value } \\
\left(X^{2}\right)^{a}\end{array}$ & $\begin{array}{l}\text { Adjusted } \\
p \text { value }^{\mathrm{b}}\end{array}$ & $\begin{array}{l}\text { OR } \\
(95 \% \\
\mathrm{Cl})^{b}\end{array}$ \\
\hline A663G & $A$ & $\begin{array}{l}39 \\
(6.34)\end{array}$ & $\begin{array}{l}38 \\
(8.43)\end{array}$ & 0.231 & 0.141 & $\begin{array}{l}1.451 \\
(0.884- \\
2.381)\end{array}$ \\
\hline $8281-8289 d^{C}$ & $\mathrm{~B}$ & $\begin{array}{l}118 \\
(19.19)\end{array}$ & $\begin{array}{l}78 \\
(17.29)\end{array}$ & 0.472 & 0.202 & $\begin{array}{l}0.796 \\
(0.561- \\
1.130)\end{array}$ \\
\hline $\mathrm{C} 5178 \mathrm{a}^{\mathrm{c}}$ & $\mathrm{D}$ & $\begin{array}{l}176 \\
(28.62)\end{array}$ & $\begin{array}{l}85 \\
(18.85)\end{array}$ & 0.000 & 0.001 & $\begin{array}{l}0.590 \\
(0.428- \\
0.814)\end{array}$ \\
\hline A249d/T6392C/G10310A & $\mathrm{F}$ & $\begin{array}{l}129 \\
(20.98)\end{array}$ & $\begin{array}{l}66 \\
(14.63)\end{array}$ & 0.008 & 0.020 & $\begin{array}{l}0.654 \\
(0.457- \\
0.936)\end{array}$ \\
\hline A4833G & G & $\begin{array}{l}16 \\
(2.60)\end{array}$ & $\begin{array}{l}29 \\
(6.43)\end{array}$ & 0.003 & 0.015 & $\begin{array}{l}2.336 \\
(1.179- \\
4.608)\end{array}$ \\
\hline T9824C & M7 & $\begin{array}{l}33 \\
(5.37)\end{array}$ & $\begin{array}{l}36 \\
(7.98)\end{array}$ & 0.101 & 0.390 & $\begin{array}{l}0.795 \\
(0.472- \\
1.341)\end{array}$ \\
\hline A4715G & M8 (M8a + C + Z) & $\begin{array}{l}36 \\
(5.85)\end{array}$ & $\begin{array}{l}53 \\
(11.75)\end{array}$ & 0.001 & 0.005 & $\begin{array}{l}2.033 \\
(1.242- \\
3.322)\end{array}$ \\
\hline T3394C & M9 & $\begin{array}{l}18 \\
(2.93)\end{array}$ & $\begin{array}{l}28 \\
(6.21)\end{array}$ & 0.010 & 0.002 & $\begin{array}{l}3.040 \\
(1.522- \\
6.061)\end{array}$ \\
\hline T489C/C10400T/T14783C/G15043A & $\begin{array}{l}\mathrm{M}^{*} \\
(\mathrm{M} 2+\mathrm{M} 10+\mathrm{M} 11+\mathrm{M} 13 \mathrm{a}+\mathrm{M})\end{array}$ & $\begin{array}{l}32 \\
(5.20)\end{array}$ & $\begin{array}{l}17 \\
(3.77)\end{array}$ & NA & & \\
\hline G5417A/C16257a/C16261T & N9a & $\begin{array}{l}11 \\
(1.79)\end{array}$ & $\begin{array}{l}19 \\
(4.21)\end{array}$ & 0.023 & 0.018 & $\begin{array}{l}2.890 \\
(1.199- \\
6.993)\end{array}$ \\
\hline T1391C/T16311C & $\mathrm{R} 1$ & $7(1.14)$ & $\begin{array}{l}2 \\
(0.44)\end{array}$ & 0.316 & 0.358 & $\begin{array}{l}0.460 \\
(0.088- \\
2.410)\end{array}$ \\
\hline
\end{tabular}

a, $\chi^{2}$-Test or Fisher's exact test. Bonferroni corrected $p<0.05 / \mathrm{n}(\mathrm{n}=15)$.

b , adjusted $p$ value and ORs (95\% Cls) determined by multivariate logistic regression analysis, adjusted for age, gender, smoking and diseases (including cardiopulmonary diseases, diabetes, obesity and hypertension determined through clinical and radiographic examinations).

c, the letter "d" indicates nucleotide deletion, the letter "a" indicates nucleotide transversion.

\section{Figures}




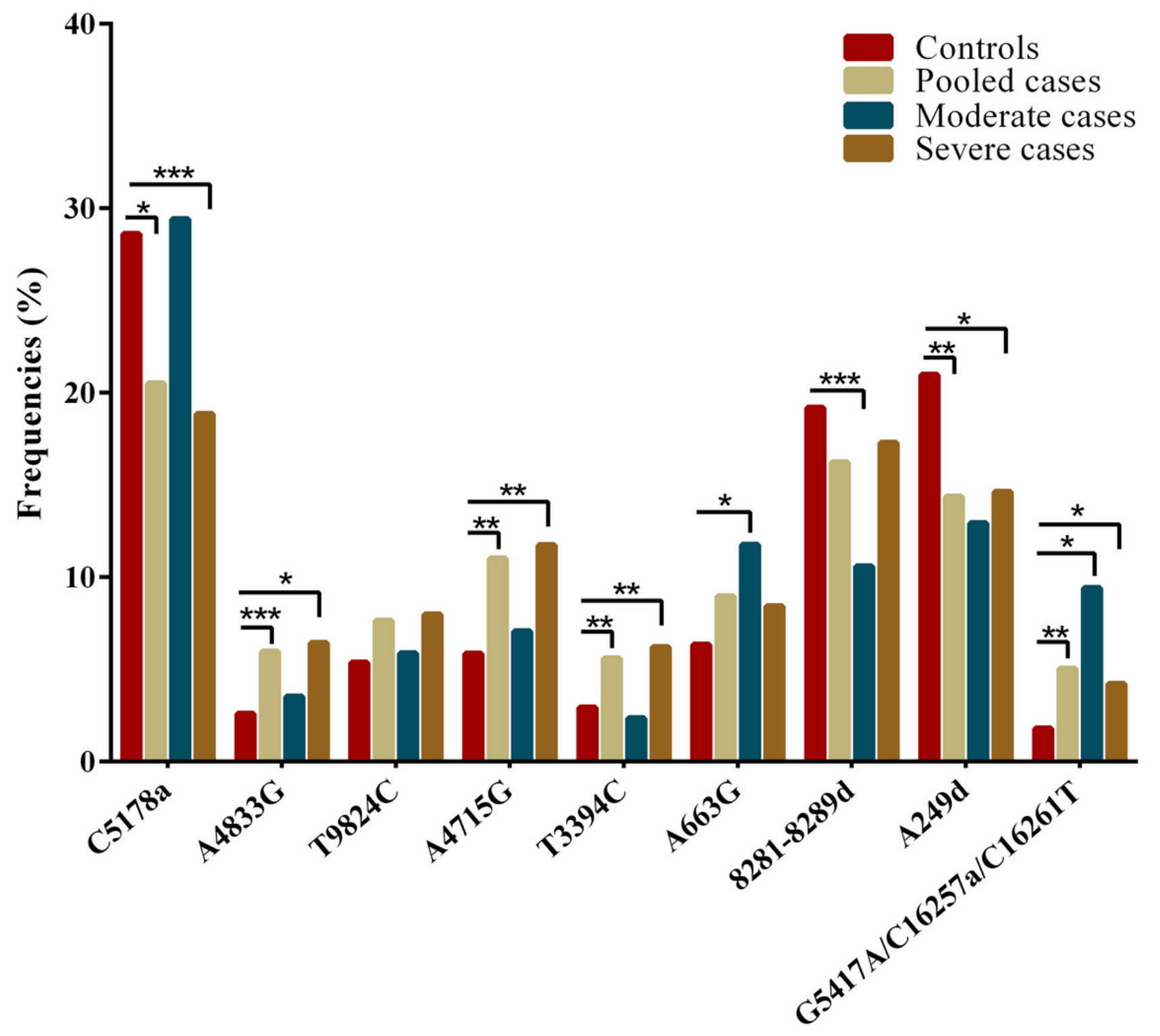

MtDNA variations defining mtDNA haplogroups

Figure 1

The frequencies of mtDNA variations defining mtDNA haplogroups in controls and COVID-19 patients. Significant differences between the two groups are labeled as follows: ${ }^{*}<<0.05,{ }^{*} p<0.01,{ }^{* \star} p<0.001$ (adjusted $p$ value was determined by multivariate logistic regression analysis, adjusted for age, sex, smoking and diseases [including cardiopulmonary diseases, diabetes, obesity and hypertension]).

\section{Supplementary Files}

This is a list of supplementary files associated with this preprint. Click to download.

- SupplementaryTable1.docx

- SupplementaryTable2theHGVSvalidationfile.docx 